\section{Infection Prevention in Transfusion Practice}

To the Editor-Contamination prevention of blood products is a challenge in transfusion practice. Contamination can occur at any point in the transfusion chain, including collection, processing, storage, issue, and administration. The objective of this communication is to describe strategies for preventing transfusion transmitted infections (TTIs) as practiced in a blood bank in a 167-bed oncology center in India.

The blood bags used for collection comply with ISO 3826 standards. ${ }^{1}$ We use bags with an attached diversion pouch for collection of the initial $20-25 \mathrm{~mL}$ of blood. This reduces bacterial contamination during collection by preventing the skin core from entering into the collection bag. The packet and blood bag are inspected for any evidence of visible contamination.

Blood collection at our center is either for component preparation or as an apheresis (ie, single-donor platelets). The cleaning and disinfection methods before blood collection are followed as per World Health Organization guidelines. ${ }^{2}$ This procedure consists of washing the donor's relevant arm with soapy water and disinfecting the anticubital fossa, initially with isopropyl alcohol for 30 seconds over a diameter of $8 \mathrm{~cm}$ in concentric manner, followed by application of $2.5 \%$ chlorhexidine with $70 \%$ isopropyl alcohol. Until screening tests are completed, whole blood is stored between 2 and $6^{\circ} \mathrm{C}$, and after component preparation, which is done within 1-2 hours of blood collection, platelets are stored at $22^{\circ} \mathrm{C}$. Packed red blood cells (RBCs) are stored between 2 to $6^{\circ} \mathrm{C}$, and plasma is stored at $-40^{\circ} \mathrm{C}$. The shelf-lives of stored products are defined as follows: 5 days for platelets, 1 year for plasma, and 6 weeks for RBCs.

Blood components can potentially transmit several pathogens, and all donated units are screened for transfusion transmissible infections (TTIs). In India, mandatory testing for TTI is required for only 5 pathogens: human immunodeficiency virus (HIV), hepatitis B virus (HBV), hepatitis C virus (HCV), malaria, and syphilis. However, the list of potentially transmissible infections is large and includes other viruses: human T-lymphotropic virus (HTLV), cytomegalovirus (CMV); bacteria (eg, Yersinia and Serratia); parasites (eg, Babesia, Trypanosoma, and Leishmania); and prion agents (eg, Creutzfeldt-Jakob disease [CJD). ${ }^{3,4}$ In our center, the Vitros ECi system (Johnson \& Johnson) is used to test for $\mathrm{HBV}$ surface antigen (HBsAg), anti-HCV antibodies, anti-HIV antibodies; immunochromatographic test (ie, a pan malaria card) is used to test for malaria (Plasmodium vivax and $P$. falciparum) antigen, and syphilis serology is performed using a card agglutination test (Rapid Plasma Reagin test). Blood units found to be serologically reactive for any of the markers (Table 1) are immediately identified, segregated, and discarded. All seroreactive donors are notified, as counseling is essential. Our data show that donor response for counseling after notification is only $\sim 9.5 \%$. Regular, repeat, voluntary donors represent a safer source of blood because they have been repeatedly tested and have low seroprevalence. The cost of serological screening for TTI in our center is Indian rupees (Rs) $180(\sim 3)$ per component or Rs $540(\sim \$ 9)$ per donation.

Quality control of blood products for content is undertaken, and $1 \%$ of all units are collected, or 4 units/month, whichever is greater. Data on sterility testing in our center show that $3.2 \%$ of RBCs, $5.2 \%$ of platelets, and $12 \%$ of fresh-frozen plasma samples were culture positive (Table 1). Most of these organisms were environmental contaminants and low-pathogenicity organisms. These findings correlate well clinically, as no septic transfusion reactions were recorded during this period (September 2013August 2014). The relatively higher rate of microbiology culture positivity could be due to more sensitive methods of detection (eg, pediatric BacTALERT blood culture bottles were used), to period of incubation (5 days vs 2 days, as practiced in some centers), and to relative volume of inoculum used $(0.5 \mathrm{~mL}$ of blood product in $30 \mathrm{~mL}$ of broth). The cost of sterility testing (bacteria and yeasts) is Rs $240(\sim \$ 4)$ per sample.

Screened blood products can be infectious, which can be due to window-period positivity (ie, nucleic acid positive while serologically nonreactive), to mutant strains of virus, to immuned silent donors (ie, immunoglobulin-deficient status), to testing errors, to agents not tested, or to unknown agents. Nucleic acid amplification tests (NATs) have further reduced the chance of TTIs. ${ }^{5}$ In centers where the NAT technique is used for screening donated units, the estimated residual risk is 1 in 360,000 for HBV, 1 in 4.3 million for HIV, and 1 in 10 million for HCV. ${ }^{3}$ Pathogen inactivation procedures (eg, Mirasol) represent a new technology used to reduce TTIs. ${ }^{4}$

Today, bacterial infections remain the most common TTI, and the risk is greatest with platelet components. Incidence of transfusion-associated septic events is reported to be 1 in 250,000 for packed RBCs but 1 in 25,000 for platelets. ${ }^{5,6}$ Bacterial contamination reactions are second only to $\mathrm{ABO}$ incompatibility as cause of death post transfusion. ${ }^{7}$ Transfusion-associated septic reactions are often underrecognized unless they are dramatic, such as shaking chills with hypotension, disseminated intravascular coagulation, or shock. If any such event occurs, transfusion must be stopped immediately, the relevant unit must be sent for culture, and the satellite units must be cultured and quarantined. Sources of bacterial contamination include asymptomatic donor bacteremia, contamination during phlebotomy, and bag contamination during processing, storage, thawing, or administration. Fresh-frozen plasma bags stored at $-40^{\circ} \mathrm{C}$ can harbor invisible fractures or micropunctures due to inappropriate handling.

Prevention of TTIs, including bacterial contamination, requires a comprehensive approach throughout the transfusion chain and the implementation of high-quality practices. Quality standards are necessary not only to fulfill legal requirements but also to ensure patient safety. 
t A в LE 1. Seroreactivity in Donors and Microbiological Culture Positivity Rate in Blood Products at Tata Medical Center, Kolkata, India

Seroreactivity in donors (September 2013-August 2014)

Anti-HIV: 13 of $6,900(0.19 \%)$

HBsAg: 47 of $6,900(0.68 \%)$

Anti-HCV: 55 of 6,900 (0.79\%)

RPR test for syphilis: 12 of $6,900(0.17 \%)$

Malaria antigen test: 0 of $6,900(0 \%)$

Microbiological culture positive rate in blood products

Packed RBCs: 2 of 31 (6.45\%); Staphylococcus warneri, Sphingomonas paucimobilis

(September 2013-August 2014)

Platelets: 1 of 46 (2.1\%); Micrococcus luteus

Fresh-frozen plasma: 2 of 15 (13.3\%); S. warnerii, S. epidermidis

NOTE. HIV, human immunodeficiency virus; HBsAg, hepatitis B surface antigen; HCV, hepatitis C virus; RPR, rapid plasma reagin;

RBC, red blood cells.

\section{ACKNOWLEDGMENT}

We gratefully acknowledge the assistance of the technologists of the Departments of Transfusion Medicine and Microbiology.

Financial support: No financial support was provided relevant to this article.

Potential conflicts of interest: All authors report no conflicts of interest relevant to this article.

\section{Sabita Basu, MD, MNAMS; ${ }^{1}$ Gaurav Goel, $\mathrm{MD}{ }^{2}$ Sanjay Bhattacharya, MD, DNB, DipRCPath, FRCPath ${ }^{3}$}

Affiliations: 1. Senior Consultant, Department of Transfusion Medicine, Tata Medical Center, Kolkata, India; 2. Junior Consultant, Department of Microbiology, Tata Medical Center, Kolkata, India; 3. Senior Consultant, Department of Microbiology, Tata Medical Center, Kolkata, India.

Address all correspondence to Dr. Sanjay Bhattacharya, MD, DNB, DipRCPath, FRCPath, CCT (UK), Consultant Microbiologist, Tata Medical Center, 14 Major Arterial Road (E-W),New Town, Rajarhat, Kolkata 700 156, India (drsanjay1970@hotmail.com).

Infect Control Hosp Epidemiol 2015;36(5):613-614

(C) 2015 by The Society for Healthcare Epidemiology of America. All rights reserved. 0899-823X/2015/3605-0024. DOI: 10.1017/ice.2015.29

\section{REFERENCES}

1. Plastic collapsible containers for human blood and blood components-part 3. Blood bag systems with integrated features. ISO 3826-3. International Organization for Standardization website. www.iso.org. Published 2006. Accessed on August 10, 2014.

2. WHO. guidelines on drawing blood: best practices in phlebotomy. Geneva, Switzerland: World Health Organization, 2010.

3. Seed CR, Kiely P, Keller AJ. Residual risk of transfusion transmitted human immunodeficiency virus, hepatitis B virus, hepatitis $\mathrm{C}$ virus and human T lymphotrophic virus. Intern Med J 2005;35(10):592-598.

4. Kwon SY, Kim IS, Bae JE, Kang JW, Cho YJ, Cho NS, Lee SW. Pathogen inactivation efficacy of Mirasol PRT System and Intercept Blood System for non-leucoreduced platelet-rich plasma-derived platelets suspended in plasma. Vox Sang 2014; 107(3):254-260.

5. Busch MP, Kleinman SH, Nemo GJ. Current and emerging risks of blood transfusions. JAMA 2003;289:959-962.
6. Agarwal N, Chatterjee K, Coshic P, Borgohain M. Nucleic acid testing for blood banks: an experience from a tertiary care centre in New Delhi, India. Transfus Apher Sci 2013;49(3):482-484.

7. Brecher ME, Hay SN. Bacterial contamination of blood components. Clin Microbiol Rev 2005;18(1):195-204.

\section{Infectious Complications Following Transrectal Ultrasound-guided Prostate Biopsy: A Canadian Tertiary Cancer Center Experience}

To the Editor-Transrectal ultrasound (TRUS)-guided prostate biopsy, used to diagnose prostate cancer, is associated with infectious complications ranging from simple cystitis to severe sepsis. ${ }^{1}$ Antimicrobial prophylaxis prior to TRUSguided prostate biopsy, most commonly using ciprofloxacin, has been shown to reduce infectious complications. ${ }^{2}$ Recent reports, however, have documented increasing rates of infections complicating TRUS-guided prostate biopsy, including infections secondary to fluoroquinolone-resistant Escherichia coli. ${ }^{3}$

We reviewed the temporal trends of infectious complications following TRUS-guided prostate biopsy at Princess Margaret Cancer Center, a 130-bed Canadian tertiary-care cancer center in Toronto, Canada, where ciprofloxacin prophylaxis is routinely prescribed prior to these procedures. Passive surveillance for complications following TRUS-guided prostate biopsy has been conducted since 2003. Following biopsy, patients are provided with both written and verbal instructions to return to the emergency department if they develop complications including fever, dysuria, or hematuria within 1 week of biopsy and to contact their urologist. Additionally, at the bottom of each computer-generated procedure report sent to the referring physician, the following message appears: "Please let us know if the patient has any late complications." 